\title{
Meningiomas with Hemorrhagic Onset: A New Bleeding Classification and its Clinical Significance
}

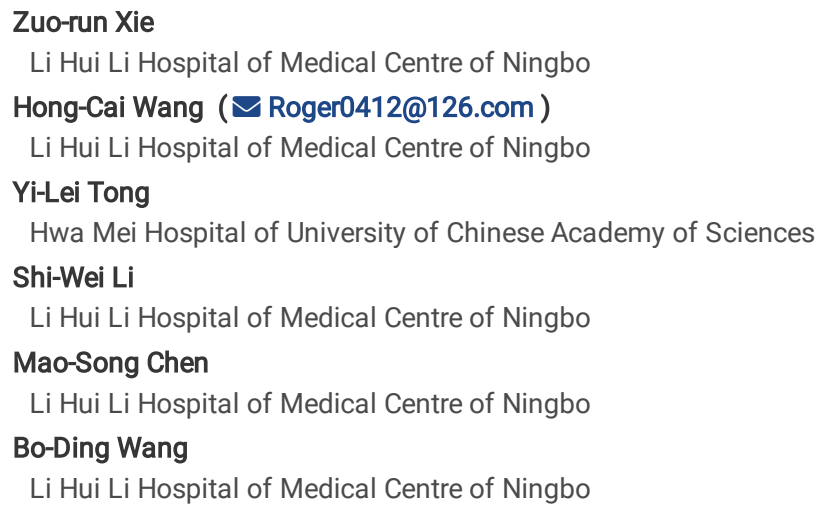




\section{Abstract}

Meningiomas are the commonest benign intracranial tumors and frequently present with a gradual onset of neurological deficits; however, their acute presentation with hemorrhagic onset appears to be a rare event. Nonetheless, because the mainstay of treatment is early surgical evacuation, prompt diagnosis of this rare category of intracranial hemorrhage is imperative. The purpose of this single-center study was to investigate the radiological characteristics and proposed a new bleeding classification for guiding diagnosis and treatment. A total of 19 consecutive patients diagnosed with hemorrhagic meningioma were enrolled in this retrospective study. Intracranial extra-axial mass, tumor-associated hemorrhage, and peritumoral brain edema were the three main radiological features. The site of tumor-associated hemorrhage included peritumoral space, subarachnoid space, subdural space, brain parenchyma, and/or intratumor. Based on the anatomical relationship between meningioma and hematoma, the spontaneous hemorrhage stemming from meningiomas were further summarized into three bleeding patterns involving purely intratumoral hemorrhage (type I), purely extratumoral hemorrhage (type II), and combined intra/extratumoral hemorrhage (type III); moreover, the type III hemorrhage usually came from the type I bleeding that extended into the surrounding regions. The symptoms in type I patients were generally mild, and early surgery was performed following adequate preoperative evaluations; the symptoms in type II patients were sometimes mild and may be sometimes moderate to severe, so early or emergency surgery was chosen according to the patients' clinical statuses; almost all type III patients had moderate to severe symptoms, and these patients usually needed emergency surgery. In addition, the patients with different bleeding types may have different pathological mechanisms underlying the bleeding. Apart from being convenient for diagnosis, this concise and practical bleeding classification could offer some implications for the treatment strategy and facilitate understanding of the associated mechanisms.

\section{Introduction}

Meningiomas are most often slow-growing intracranial tumors and usually present with symptoms such as headache, dizziness, seizures, or the gradual progression of neurological deficits; however, their acute presentation with spontaneous hemorrhage appears to be a rare event ${ }^{1-3}$. Although rare, intracranial hemorrhage stemming from meningiomas could have a dramatic effect on outcomes and may even be an apparent life-threatening event due to acutely increased intracranial pressure ${ }^{2-4}$. While no difficulty is encountered in the diagnosis of hemorrhage in a previously known brain tumor, a hemorrhagic onset as the initial presentation of a brain tumor may pose some diagnostic problems.

Therefore, it is the key point of early diagnosis and correct treatment to improve their outcomes. Some clinical features of such a condition have been characterized; however, previous studies regarding this condition were limited to single-case reports or small case series ${ }^{2-6}$. These cases in literatures were reported according to the site of hemorrhage such as subarachnoid space, subdural space, peritumoral space, brain parenchyma, or intratumor. Unfortunately, this description of tumoral bleeding cannot reflect the relationship between meningioma and hemorrhage; besides, it also has no practical significance for clinical diagnosis and contributes little to guiding treatment. Herein, we proposed a new classification system of hemorrhage associated with meningiomas that was based on a retrospective analysis of 19 cases, and explored its clinical significance.

\section{Materials And Methods Study population.}

We confirm that all methods were performed in accordance with the relevant guidelines and regulations. Following Institutional Ethics Board of Li Hui Li Hospital of the Medical Centre of Ningbo approval, this retrospective study enrolled a total of 649 consecutive patients with meningiomas who underwent craniotomy for tumor resection at the Li Hui Li Hospital of the Medical Centre of Ningbo between July 2008 and March 2021. Nineteen of these 649 patients presented with acute spontaneous hemorrhage stemming from meningiomas. Twenty cases with nonhemorrhagic meningiomas were chosen randomly in the same period and served as a comparison group (group 4). The clinical data including age, gender, onset symptoms, Simpson resection grade, and histological variants, were extracted from the medical records. The data of pre- and postoperative computed tomography (CT), magnetic resonance imaging (MRI), and/or CT angiography (CTA) were examined via PACS. Patients with evidence of bleeding tendency or other predisposing factors for hemorrhage, such as receipt of antiplatelet or anticoagulant medication, were excluded from this study.

\section{Radiological evaluation.}

Radiological outcomes were analyzed by CT scans, MRI, and/or CTA. The radiological features, including tumor location, size, signal intensity, contrast enhancement, tumor margins, and hemorrhagic site, were analyzed independently by two neurosurgeons. Tumor size was determined by measuring the greatest diameter of the enhanced tumor on the preoperative CT or the contrast enhancement of T1-weighted MRI. The signal intensities of tumors on T2weighted MRI were categorized as either hypointense, isointense, or hyperintense relative to that of the cortical gray matter on the same MRI. Peritumoral brain edema (PTBE) was also evaluated on T2-weighted MRI and the extent of PTBE was assessed by calculation of the edema index (EI). The EI was calculated in this study according to the methodology used in previous studies ${ }^{7}$.

In order to further reflect the relationship between meningioma and hemorrhage, a new bleeding classification system of meningiomas was proposed and such 19 cases were correspondingly divided into three subgroups (Fig. 1): the type I bleeding was defined as the hemorrhage being purely found in tumor (group 1); the type II bleeding was referred to the purely extratumoral hemorrhage and the hemorrhage may be found in the peritumoral, subdural, subarachnoid, or/and intracerebral spaces (group 2); the type III bleeding manifested both intra- and extratumoral hemorrhage (group 3). 


\section{Treatment and prognostic assessment.}

Hematoma evacuation and a macroscopically complete resection of the tumor were planned to perform in one stage for all patients. Treatment strategy was carried out according to the patients' clinical statuses. The Simpson resection grade classification was used to evaluate the extent of resection on the basis of the surgical reports and the postoperative MRI. Pathological grading of the tumors was according to the 2007 WHO classification criteria. Follow-up data were collected from clinical visits and telephone interviews, with a mean follow-up time of 48.8 months (standard deviation = 35.13, range 3-125). The patients' postoperative statuses were investigated by physical examination and enhanced MRI. Radiological and functional assessments were usually performed preoperatively, at discharge, at 6 months postoperatively, and annually thereafter.

\section{Statistical methods.}

All continuous data were expressed as the mean \pm standard deviation (SD). Statistical analysis was performed with the SAS system (version 8.1 , Cary, North Carolina, USA), while the statistical image was drawn by R software (Version 3.5.3). Patients' age, El, and tumor size among the four groups were compared using one-way analysis of variance (ANOVA) followed by Newman-Keuls post-hoc tests. Qualitative data were compared by the Chi-square test, and the Fisher's exact test method was used to calculate the exact probability. Differences were considered to be significant at $p<0.05$.

\section{Results}

\section{Clinical features.}

Of the 649 meningioma patients treated, 19 (2.9\%) presented with hemorrhagic onset. These 19 cases had complete medical records and were included in this study. Their clinical data were summarized in Table 1. There were 9 males and 10 females, yielding a male-to-female ration of 0.9:1. The confirmed age at diagnosis ranged from 19 to 79 years, and the average age was $55 \pm 14.6$ years old. The average age had no statistical difference among the four groups (Fig $2 ; \mathrm{F}=0.2401 / P>0.05)$; there was also no significant difference in the clinical factor of sex among these groups $\left(X_{2}=0.7683 / P>0.05\right)$. All the patients experienced a stroke-like episode characterized by the sudden onset of acute headache, nausea and vomiting, vertigo, epileptic seizures, hemiparesis, and/or altered consciousness. Hemorrhagic symptom was the first clinical presentation for 14 patients (73.7\%), and 5 patients (26.3\%) had initial symptoms such as mild headache, dizziness, mental disturbance, or limb weakness before bleeding events. 
Table 1

The clinical features of 19 meningiomas with hemorrhagic onset.

\begin{tabular}{|c|c|c|c|c|c|c|c|c|c|c|}
\hline Case & $\begin{array}{l}\text { Age } \\
\text { (years) }\end{array}$ & Sex & Symptoms & $\begin{array}{l}\text { Tumor } \\
\text { location }\end{array}$ & $\begin{array}{l}\text { Bleeding } \\
\text { type }\end{array}$ & Tumor subtype & $\begin{array}{l}\text { Tumor } \\
\text { size } \\
(\mathrm{mm})\end{array}$ & $\begin{array}{l}\text { Resection } \\
\text { grade }\end{array}$ & $\begin{array}{l}\text { Signal } \\
\text { intensity }\end{array}$ & Outcome \\
\hline 1 & 59 & $\mathrm{~F}$ & $\begin{array}{l}\text { Headache/ hemiparesis } \\
\text { /aphasia }\end{array}$ & Convexity & Type I & Fibrous & 65 & Grade I & Iso- & $\begin{array}{l}\text { Alive/hemipa } \\
\text { tumor recurre }\end{array}$ \\
\hline 2 & 45 & M & Headache/ nausea/vomiting & Parasagital & Type I & Atypical & 39 & Grade II & Hyper- & $\begin{array}{l}\text { Alive/tumor } \\
\text { recurrence }\end{array}$ \\
\hline 3 & 41 & M & Headache/nausea & Convexity & Type I & Meningothelial & 22 & Grade I & Hyper- & $\begin{array}{l}\text { Alive/no tumı } \\
\text { recurrence }\end{array}$ \\
\hline 4 & 41 & M & Headache/hemiparesis & Convexity & Type I & Meningothelial & 73 & Grade I & Hyper- & $\begin{array}{l}\text { Alive/no tumı } \\
\text { recurrence }\end{array}$ \\
\hline 5 & 46 & $\mathrm{~F}$ & Headache & Parasagital & Type I & Microcystic & 31 & Grade III & Hyper- & $\begin{array}{l}\text { Alive/no tumı } \\
\text { recurrence }\end{array}$ \\
\hline 6 & 56 & $\mathrm{~F}$ & Headache/epilepsy & Convexity & Type I & Atypical & 25 & Grade I & Iso- & $\begin{array}{l}\text { Alive/no tumı } \\
\text { recurrence }\end{array}$ \\
\hline 7 & 71 & $\mathrm{~F}$ & Headache/ nausea & $\begin{array}{l}\text { Middle } \\
\text { skull base }\end{array}$ & Type I & Angiomatous & 53 & Grade IV & Hyper- & $\begin{array}{l}\text { Alive/no tumı } \\
\text { progression }\end{array}$ \\
\hline 8 & 63 & M & $\begin{array}{l}\text { Headache/vertigo/facial } \\
\text { paralysis }\end{array}$ & CPA & Type I & Fibrous & 25 & Grade II & Нypo- & $\begin{array}{l}\text { Alive/facial } \\
\text { paralysis/no } \\
\text { recurrence }\end{array}$ \\
\hline 9 & 57 & $\mathrm{~F}$ & Headache/nausea & Convexity & Type II & Fibrous & 43 & Grade I & Iso- & $\begin{array}{l}\text { Alive/no tumı } \\
\text { recurrence }\end{array}$ \\
\hline 10 & 71 & M & $\begin{array}{l}\text { Headache/drowsy/ } \\
\text { hemiparesis }\end{array}$ & Convexity & Type II & Malignant & 57 & Grade I & Hyper- & $\begin{array}{l}\text { Alive/Hemipa } \\
\text { tumor recurre }\end{array}$ \\
\hline 11 & 46 & $\mathrm{~F}$ & Headache & Parasagital & Type II & Angiomatous & 21 & Grade II & Hyper- & $\begin{array}{l}\text { Alive/no tumı } \\
\text { recurrence }\end{array}$ \\
\hline 12 & 71 & M & Coma/epilepsy & $\begin{array}{l}\text { Anterior } \\
\text { skull base }\end{array}$ & Type II & Atypical & 68 & Grade III & / & $\begin{array}{l}\text { Alive/ epileps } \\
\text { recurrence }\end{array}$ \\
\hline 13 & 53 & $\mathrm{~F}$ & Headache/nausea/vomiting & Parafalx & Type II & Psammomatous & 51 & Ggrade I & Нypo- & $\begin{array}{l}\text { Alive/no tumı } \\
\text { recurrence }\end{array}$ \\
\hline 14 & 19 & M & Headache/drowsy & Convexity & Type III & Meningothelial & 45 & Grade I & Hyper- & $\begin{array}{l}\text { Alive/no tumı } \\
\text { recurrence }\end{array}$ \\
\hline 15 & 79 & M & Coma & Parasagital & Type III & Malignant & 68 & Grade I & / & Died \\
\hline 16 & 51 & $\mathrm{~F}$ & $\begin{array}{l}\text { Headache/drowsy/ } \\
\text { hemidysesthesia }\end{array}$ & Convexity & Type III & Transitional & 52 & Grade I & Hyper- & $\begin{array}{l}\text { Alive/no tumı } \\
\text { recurrence }\end{array}$ \\
\hline 17 & 53 & $\mathrm{~F}$ & Headache/drowsy/hemiparesis & Convexity & Type III & Angiomatous & 55 & Grade I & Hyper- & $\begin{array}{l}\text { Alive/no tumı } \\
\text { recurrence }\end{array}$ \\
\hline 18 & 64 & M & $\begin{array}{l}\text { Headache/ } \\
\text { hemiparesis }\end{array}$ & Parasagital & Type III & Fibrous & 41 & Grade II & Iso- & $\begin{array}{l}\text { Alive/no tumı } \\
\text { recurrence }\end{array}$ \\
\hline 19 & 39 & $\mathrm{~F}$ & Headache/nausea/vomiting & Parafalx & Type III & Secretory & 31 & Grade I & Hyper- & $\begin{array}{l}\text { Alive/no tumı } \\
\text { recurrence }\end{array}$ \\
\hline
\end{tabular}

\section{Radiological characteristics.}

All 19 patients had preoperative CT scans, 17 patients had preoperative MRI, and 7 patients had preoperative CTA in our PACS database. Preoperative MRI was not performed in two cases with respective type II and type III bleeding due to severe symptoms. All patients had postoperative CT and MRI scans. Preoperative CT and MRI generally gave evidence of well-defined, dense, contoured extra-axial masses displacing the adjacent brain and acute or subacute hemorrhage associated with masses. Abnormal blood vessels, such as aneurysms or arteriovenous malformations, were not detected on CTA. According to the new bleeding classification, 8 masses manifested the type I bleeding (Fig. 3), 5 masses present with the type II bleeding (Fig. 4), and 6 masses were the type III bleeding (Fig. 5). Moreover, the type I bleeding could penetrate the tumor and result in type III bleeding.

On MRI with gadolinium enhancement, the mass with a dural base showed moderate to strong enhancement except for the portion representing hemorrhage. All cases in group 2 were homogenous enhancement and the other cases were heterogeneous enhancement. Four masses in group 3 (80\%), 5 masses in group 1 (62.5\%), and 2 masses in group 2 (50\%) presented hyperintensity on T2-weighted images. Although the occurrence rate of hyperintensity on T2weighted images had no significant difference among the four groups $\left(X_{2}=0.9681 / P>0.05\right)$, the patients in group 3 had the highest rate. 
All masses in group 2 and group 3 showed PTBE on T2-weighted images; 4 masses in group 1 (50\%) showed PTBE on T2-weighted images and this occurrence rate was similar to it of the control group $\left(X_{2}=0.0036 / P>0.05\right)$. The El was $3.12 \pm 0.97$ in group 3 and $2.84 \pm 0.59$ in group 2 , which were both significantly higher than those in group 1 and control group (Fig. $6 ; P<0.05)$. The El between group 3 and group 2 had no significant difference $(P>0.05)$; there was also no statistical difference between group 1 and control group $(P>0.05)$. The hemorrhagic masses located at the convexity in 9 patients (47.3\%), in the parasagittal areas in 5 patients $(26.3 \%)$, in the parafalx in 2 patients, at the skull base in 2 patients, and in the cerebellopontine angle in 1 patient; the convexity and parasagittal areas were the two most common sites for hemorrhagic meningiomas. The tumor size ranged from 2.1 to $7.3 \mathrm{~cm}$ (mean, $4.6 \pm 1.7 \mathrm{~cm}$ ), with 10 tumors $(52.6 \%)<5.0 \mathrm{~cm}$ and 7 tumors $(47.4 \%)>5.0 \mathrm{~cm}$ in diameter. There was no statistical difference with respect to the tumor size among the four groups (Fig. $7 ; \mathrm{F}=0.4163 / P>0.05$ ).

\section{Intraoperative findings.}

The symptoms in most patients of group 1 (7/8 cases; $87.5 \%)$ were mild and their clinical statuses were stable, and selective surgeries were performed following adequate preoperative evaluations; the patients' symptoms in group 2 were sometimes mild ( $3 / 5$ cases; $60 \%)$ and may be sometimes moderate to severe ( $2 / 5$ cases; $40 \%)$, so emergency or early surgeries were chosen according to the patients' clinical statuses; almost all patients (5/6 cases; $83.3 \%$ in group 3 have moderate to severe symptoms, and these patients were performed emergency surgery after necessary examinations.

Hematoma evacuation and a macroscopically complete resection of the tumor were performed in one stage for all patients except 1 patient. During the operation, a solid extraparenchymal tumor attached to the dura and tumor-associated hemorrhage were found in every case; the surface of the brain contacting tumor was intact in 12 cases and invaded by the tumor in 7 cases ( 2 in group $1 ; 2$ in group 2; 3 in group 3 ). All tumors in group 3, 6 cases ( $75 \%$ ) in group 1 , and 2 cases (40\%) in group 2 had soft consistency. Eight masses in group 1 had only intratumoral hemorrhage, and necrotic tumor tissue was usually found; 5 masses in group 2 were only surrounded by extratumoral hemorrhage involving peritumoral hemorrhage, subarachnoid hemorrhage, subdural hemorrhage, and/or intracerebral hemorrhage; the remaining 6 masses in group 3 included both intratumoral hemorrhage and extratumoral hemorrhage, and normal vessels invaded by tumor were found in 2 masses. These operative results were consistent with the corresponding radiological presentations and the new bleeding classification (Fig. 8). Simpson classification in hemorrhagic masses were classified as grade I $63.1 \%(n=12)$, grade II $21.1 \%(n=4)$, grade III $10.5 \%(n=2)$, and grade IV $5.3 \%(n=1)$.

\section{Pathological and surgical outcomes.}

The diagnosis of meningioma was established according to standard histopathological criteria following the WHO system; these identified tumors were subtyped as fibrous $(n=4)$, meningothelial $(n=3)$, atypical $(n=3)$, angiomatous $(n=2)$, malignant $(n=2)$, microcystic $(n=2)$, transitional $(n=1)$, psammomatous $(n=1)$, and secretory $(n=1)$; correspondingly, 14 cases were WHO Grade I tumors, 3 cases were WHO Grade II tumors, and 2 case was WHO Grade III tumor. Although there was no statistical analysis in this study, above pathological types were almost distributed in each group; endothelial hyperplasia, thin-walled dilated vessels, venous obstruction, and tumor infarction were observed more frequently on microscopic examination in group 1 and group 3 patients. Radiotherapy was administered as an adjuvant treatment in 3 patients (15.8\%) after initial surgery for 2 malignant cases and 1 case of grade IV resection. Postoperative recoveries of these patients were uneventful; 1 patient died of cardiovascular event on postoperative 7 years and the other patients are currently alive. However, the tumors in 2 patients recurred within a follow-up period of two and five years respectively, and underwent a second surgery.

\section{Discussion}

Malignant brain tumors, such as metastatic brain tumors, glioblastomas, and melanoma sometimes lead to intracranial hemorrhage ${ }^{8}$. However, spontaneous hemorrhage as an initial presentation for meningiomas, even though reported, is less frequent, although they are usually vascular tumors ${ }^{4}$. The rarity of this condition not only makes determining causative factors of the hemorrhage challenging, but also makes the mechanisms of spontaneous hemorrhage harder to understand $\mathrm{d}^{4,9,10}$. Fortunately, some sporadic cases of spontaneous hemorrhage in meningiomas have been reported and many clinical features of such a condition have been characterized.

Although CT is the reference standard for the detection of hemorrhage, this condition of meningiomas with intracranial hemorrhage is not always easy to distinguish on CT, and multi-model radiological examinations are needed ${ }^{4}$. Brain CT and MRI usually displayed well-defined, dense, contoured extra-axial masses displacing the adjacent brain along with acute or subacute hemorrhage. The site of the hematoma was generally not typical for hypertensive intracerebral bleeding. On MRI with gadolinium enhancement, the mass with a dural base showed moderate to strong enhancement except for the portion representing hemorrhage. These patients could gain good outcomes when they are managed appropriately with early diagnosis and correct treatment ${ }^{2,4}$. The majority of these cases require surgical intervention because observation and other treatments do not alleviate mass effect and risk of repeated hemorrhage $\mathrm{e}^{2,11}$. Previous reports had provided some information on the signal intensity on MRI and indicated hyperintensity on T2-weighted MRI as a risk factor of meningioma bleeding ${ }^{10,12}$; however, this finding was just found more frequently in the patients with type I and type III bleeding in this study. While the implication of this hyperintensity has not been fully established, some cases with intratumoral hemorrhage may be attributed to the tumors' soft consistency ${ }^{12-14}$.

Increased bleeding tendency was found to be associated with two age groups ( $<30$ years and $>70$ years), convexity and intraventricular locations, and fibrous meningiomas ${ }^{2}$; however, the most frequent localizations were the convexity and parasagittal areas in this study, and their pathological outcomes of these tumors in this study followed the histopathological distribution of meningiomas in general ${ }^{1}$. Although the patients with type II and type III bleeding had 
significant PTBE comparing with control group and PTBE might be an indicator for the certain bleeding types, PTBE could be just as a result of the tumor hemorrhage. The exact mechanism of the hemorrhage from meningiomas is not fully understood; however, several pathological mechanisms have been hypothesized to explain this rare condition $2,4,9-12$. The proposed mechanisms could include rupture from weakened blood vessels, endothelial proliferation and subsequent vascular occlusion, direct vascular invasion by tumor cells, accumulation of bioactive substances in tumor, concomitant vascular malformation or aneurysm, stretching and rupture of subdural bridging veins, tumor growth-induced venous compression associated with peritumoral edema, and infarction owing to rapid growth of the tumor. In addition, two distinct types of blood vessels involving differentiated and undifferentiated vessels were determined in our prior study; undifferentiated blood vessels contribute to a fragile state of tumor vasculature that can be disrupted by a precipitating event, thus leading to spontaneous hemorrhage ${ }^{4}$.

The above proposed mechanisms were mainly based on reported cases. Among these cases, they were most frequently present according to the site of hemorrhage such as subarachnoid hemorrhage, intracerebral hematoma, intratumoral hemorrhage, or subdural hematoma ${ }^{5,6,9-12}$. Unfortunately, this description cannot reflect the relationship between meningioma and hemorrhage; moreover, it contributes little to guiding clinical diagnosis and treatment. Correlating prior reports of such cases with our cases, a new bleeding classification of meningiomas was proposed on the basis of the anatomical relationship between meningioma and hematoma. We summarized this special hemorrhage from meningioma into three bleeding patterns: purely intratumoral hemorrhage, purely extratumoral hemorrhage, and combined intratumoral and extratumoral hemorrhage. In addition, intratumoral hemorrhage may penetrate the tumor and result in extratumoral hemorrhage; as a result, the type of combined hemorrhage usually came from the intratumoral bleeding that extended into the surrounding intracranial spaces.

Apart from showing the direct relationship between meningioma and hemorrhage, this new bleeding classification is easier to understand the possible mechanism of meningioma hemorrhage. For example, stretching and rupture of subdural bridging veins may explain the purely extratumoral hemorrhage involving subdural hematoma and subarachnoid hemorrhage. Rupture from weakened or undifferentiated blood vessels is usually associated with purely intratumoral hemorrhage. The extratumoral hemorrhage should be secondary to intratumoral bleeding in the third type of hemorrhage. Infarction and necrosis owing to rapid growth of the tumor could explain the third type of hemorrhage. Traumatic head injury is unlikely to be a causative factor in cases with purely intratumoral hemorrhage. In syncytial meningiomas, the bleeding is probably related to the presence of intratumoral vasoactive substances released, such as histamine, which could induce vasodilatation and result in the purely intratumoral hemorrhage. In addition, cerebral edema and venous obstruction can cause infarction and then usually induce the purely extratumoral hemorrhage.

Emergency or early one-stage total removal of the hemorrhagic meningioma and hematoma is the main treatment of choice ${ }^{2-4,10-12}$. The risks of meningioma hemorrhage usually vary with the amount of bleeding, the location of bleeding, the size of tumor, or the location of tumor. There was no difference of the therapeutic method for different bleeding patterns; however, this bleeding classification system could offer some implications for the treatment strategy. According to the review of prior cases reported in the literature and such cases in this study ${ }^{4-6,9-12,14-16}$, the symptoms in most patients with the first bleeding type are usually mild and their clinical statuses are generally stable, and early or selective surgery could be performed following adequate preoperative evaluations; the symptoms in patients with the second bleeding type are sometimes mild and may be sometimes moderate to severe, so emergency or early surgery should be chosen according to the patients' clinical status; almost all patients with the third bleeding type have moderate to severe symptoms, and these patients usually need emergency surgery after necessary examinations. Recognizing these facts and consequent treatment changes may result in improvement of patients' outcomes.

Although the data are preliminary, the study highlights the new bleeding classification of meningiomas based on the anatomical relationship between meningioma and hematoma. Moreover, sharing our opinion and typical radiographic images may help improve awareness of this special condition. Future research will focus on the issue of collecting more cases to explore the finding in this study.

\section{Conclusions}

In summary, patients with meningiomas who are being kept on follow-up have a low but definite risk of tumor associated hemorrhage. The meningioma patients with hemorrhagic onset usually experienced a stroke-like episode. Three bleeding patterns were proposed in the present study: purely intratumoral hemorrhage, purely extratumoral hemorrhage, and combined intratumoral and extratumoral hemorrhage. This bleeding classification is easier to understand the possible hemorrhagic mechanisms and could offer some implications for treatment strategy.

\section{Abbreviations}

CT: Computed tomography; MRI: magnetic resonance imaging

\section{Declarations}

\section{Authors' contributions}

ZX, HW and YT wrote the manuscript. SL and BW contributed to clinical data collection and figure design. MC revised the manuscript. HC contributed to patient management. All authors read and approved the final manuscript.

\section{Funding}


The clinical data collection in this work was supported by grant from the Natural Science Foundation of Ningbo (No. 2019A610283). The figure design and case follow-up in this work were supported by grants from the Ningbo Municipal Bureau of Science and Technology (No. 2014C50089; No. 2018A10054).

\section{Competing interests}

The authors declare that they have no competing interests.

\section{Availability of data and materials}

The datasets used and/or analyzed during the current case report are available from the corresponding author on reasonable request.

\section{Ethics approval and consent to participate}

Approval of the retrospective study was obtained from the research Ethics Committee of the Li Hui Li Hospital of the Medical Centre of Ningbo. An informed consent was obtained from all the participants.

\section{References}

1. Goldbrunner, R. et al. EANO guidelines for the diagnosis and treatment of meningiomas. The Lancet Oncology, 17, e383-912 (2016).

2. Bosnjak, R. et al. Spontaneous intracranial meningioma bleeding: clinicopathological features and outcome. J. Neurosurg, 103, 473-484 (2005).

3. Pereira, B. J. A. et al. Assessment of hemorrhagic onset on meningiomas: systematic review. Clinical Neurology and Neurosurgery.106175 (2020).

4. Wang, H. C. et al. An underlying pathological mechanism of meningiomas with intratumoral hemorrhage: undifferentiated microvessels. World neurosurgery, 94, 319-327 (2016).

5. Krisht, K. M. et al. Myxoid meningioma: a rare metaplastic meningioma variant in a patient presenting with intratumoral hemorrhage: case report. $J$. Neurosurg, 116, 861-865 (2012).

6. Kouyialis, A. T. et al. Peritumoural haematoma and meningioma: a common tumour with an uncommon presentation. J. Clin. Neurosci, 11, 906-909 (2004).

7. Bitzer, M. et al. Tumor-related venous obstruction and development of peritumoral brain edema in meningiomas. Neurosurgery, 42, 724-728 (1998).

8. Schrader, B. et al. Spontaneous intracranial haematomas caused by neoplasms. Acta neurochirurgica, 142, 979-985 (2000).

9. Kim, D. G. et al. Meningioma manifesting intracerebral haemorrhage: a possible mechanism of haemorrhage. Acta neurochirurgica, 142, 165-168 (2000).

10. Niiro, M. et al. Clinico-pathological study of meningiomas with haemorrhagic onset. Acta Neurochir (Wien), 145, 767-772 (2003).

11. Jr, S. S. et al. Meningiomas associated with hemorrhage: A report of two cases with a review of the literature. Neuropathology, 19, 150-160 (1999).

12. Lin, R. H. et al. Meningioma with purely intratumoral hemorrhage mimicked intracerebral hemorrhage: case report and literature review. Journal of Medical Sciences, 36, 158 (2016).

13. Yamaguchi, N. et al. Prediction of consistency of meningiomas with preoperative magnetic resonance imaging. Surgical neurology, 48, 579-583 (1997).

14. Kim, J. H. et al. A case of benign meningioma presented with subdural hemorrhage. Brain Tumor Res Treat, 3, 30-33 (2015).

15. Lefranc, F. et al. Intracranial meningiomas revealed by non-traumatic subdural haematomas: a series of four cases. Acta neurochirurgica, 143, $977-983$ (2001).

16. Mangubat, E. Z. et al. Major intratumoral hemorrhage of a petroclival atypical meningioma: case report and review of literature. Skull Base, 20, $469-474$ (2010).

\section{Figures}



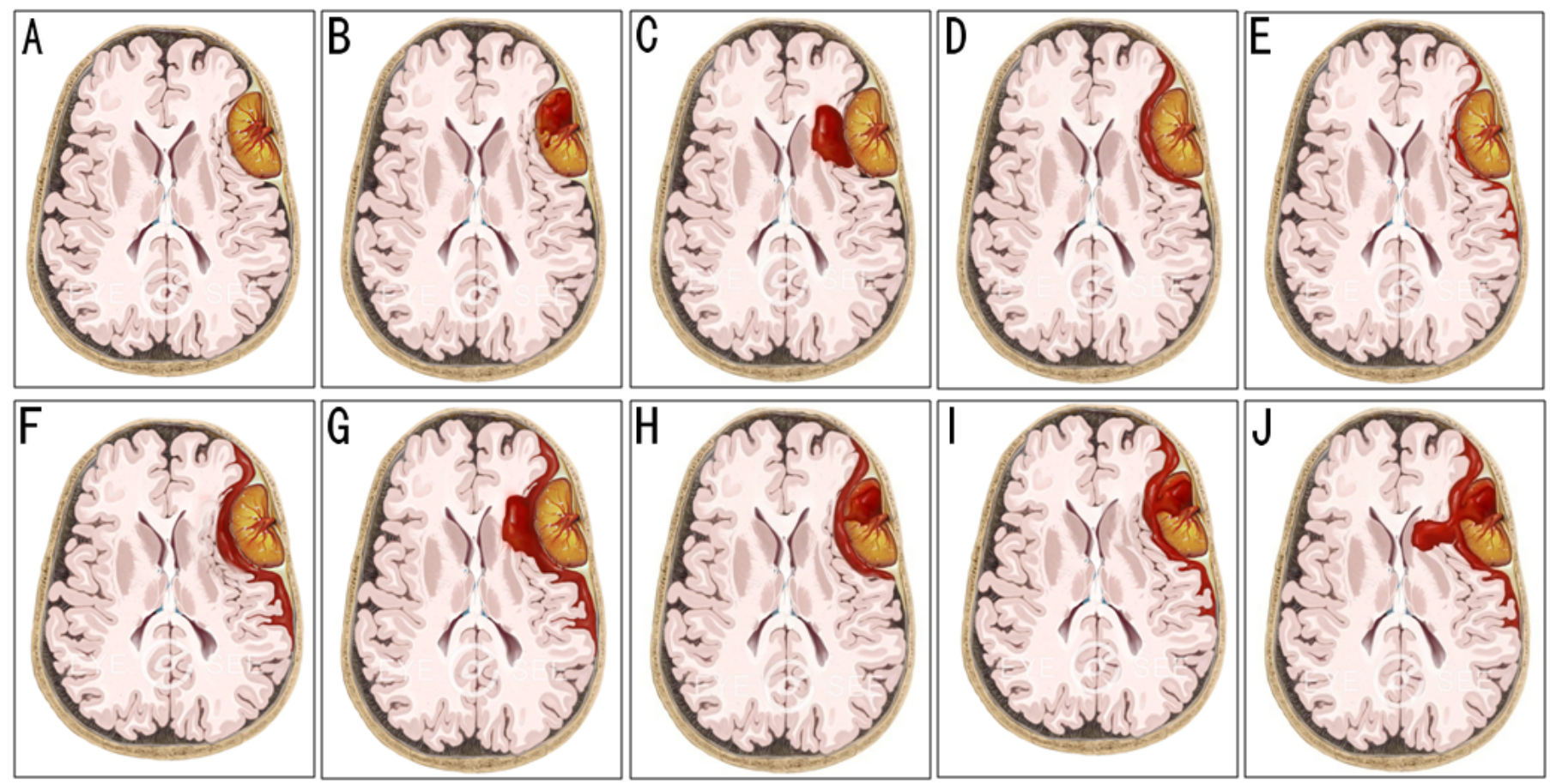

Figure 1

Illustration of different types of spontaneous hemorrhage from meningiomas. (A) A meningioma located in left temporal region. (B) The first bleeding type was purely intratumoral hemorrhage. (C-G) The second bleeding type was purely extratumoral hemorrhage. (H-J) The third bleeding type combined intratumoral hemorrhage with extratumoral hemorrhage and the extratumoral hemorrhage originated from intratumoral bleeding.

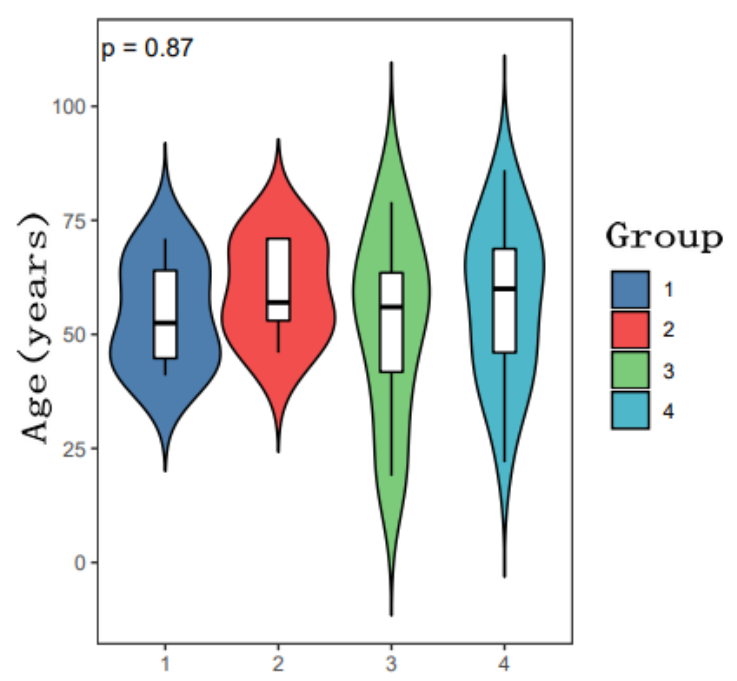

Figure 2

The graph showing the difference in average age among the four groups. The average age had no statistical difference among the four groups $(P>0.05)$. 


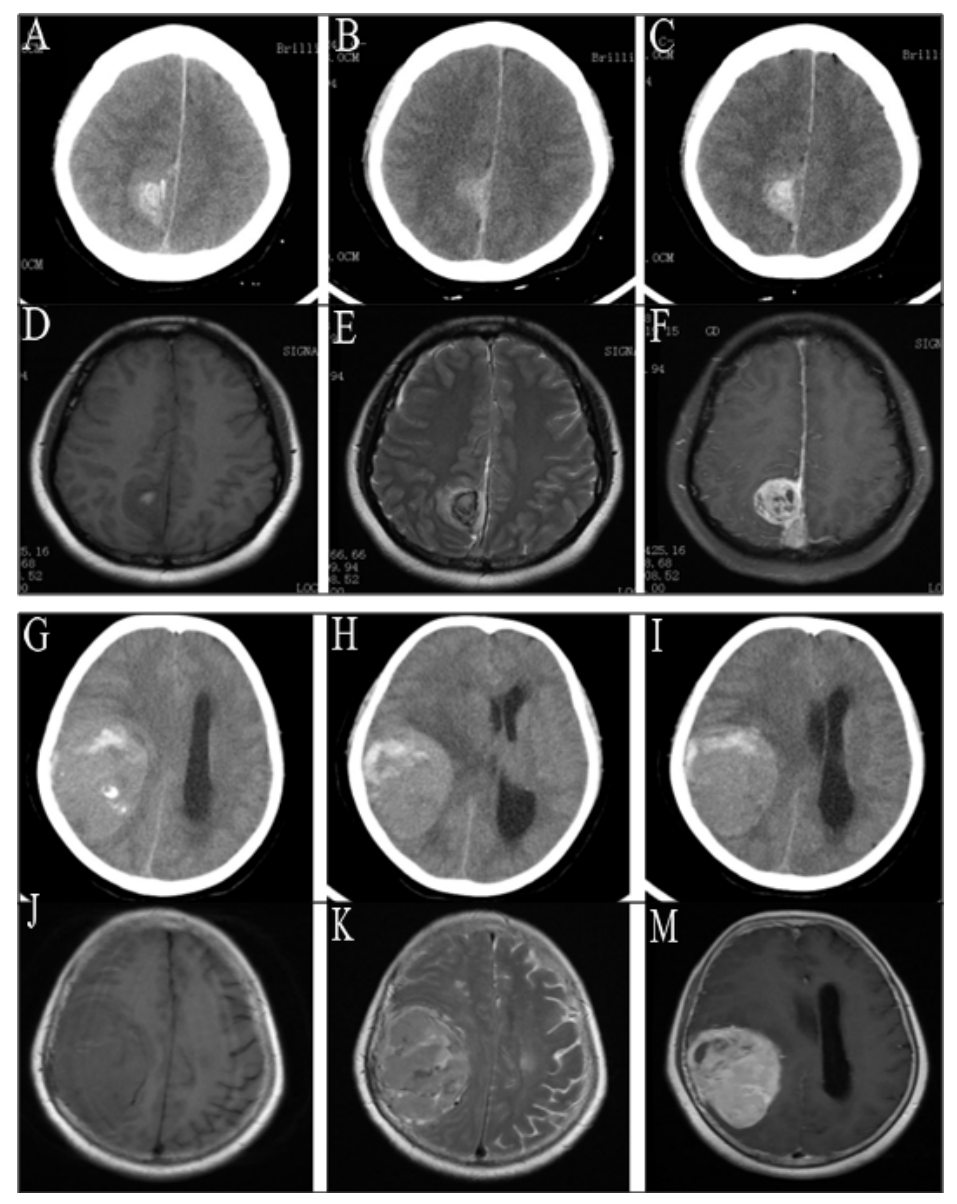

Figure 3

Radiological findings on representative case 5 and case 4 with solitary intratumoral hemorrhage. Preoperative brain CT showing a round iso-dense mass in the right parasagital area $(A-C)$ and a large mass with high density in the right parietal region (G-I) with multi-foci of hyperdensity suggestive of intratumoral hemorrhage; Brain MRI revealing two dural-based masses in the right parasagital area (D-F) and parietal region (J-M) with heterogeneous enhancement on contrast. The peripheral area of the two masses was significantly enhanced, and the central non-enhancing area suggested acute intratumoral bleeding. 

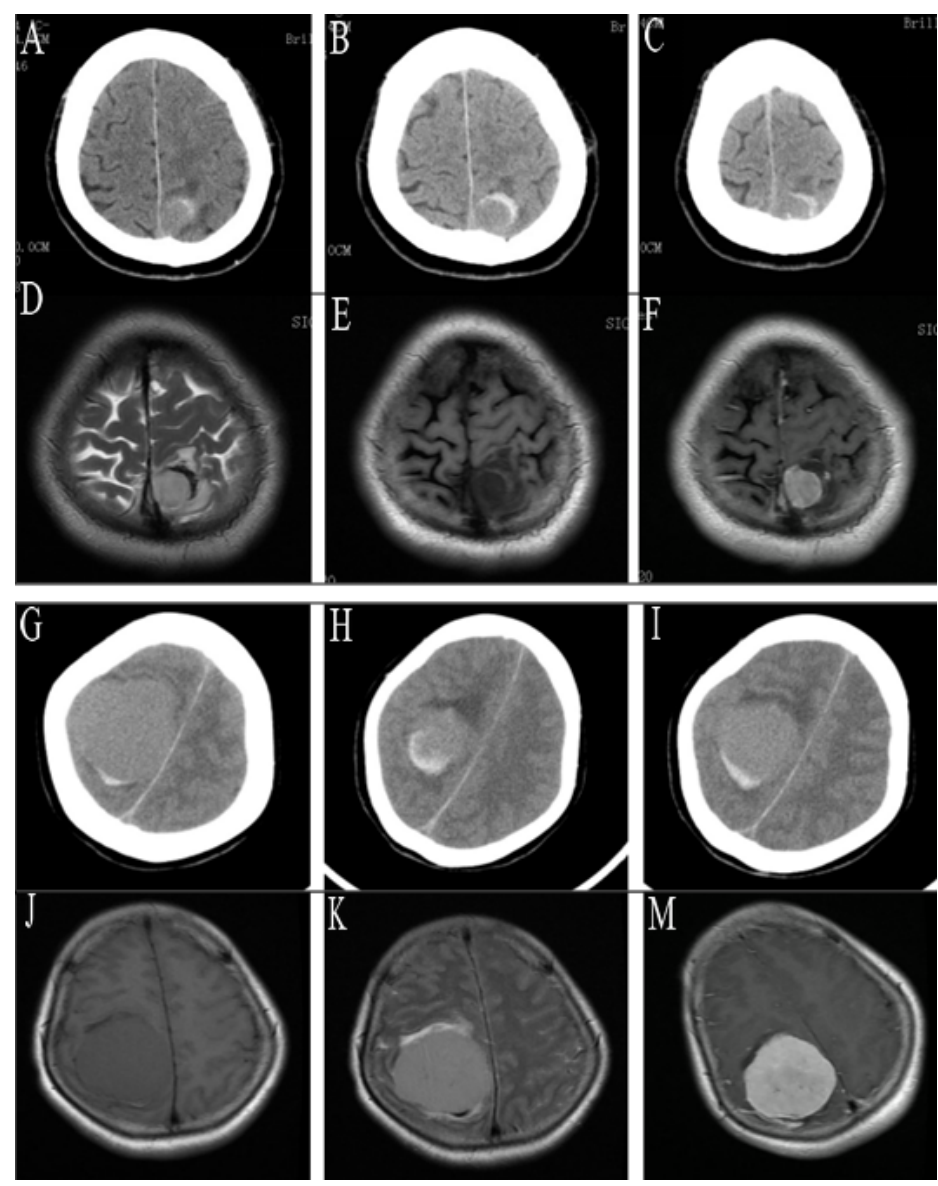

Figure 4

Neuroimaging findings on representative case 11 and case 10 with purely extratumoral hemorrhage. Preoperative brain CT and MRI showing two extra-brain masses in the left parasagital region (A-F) and right parietal area (G-M) respectively, which were homogeneously enhanced and had acute peritumoral hematoma. 

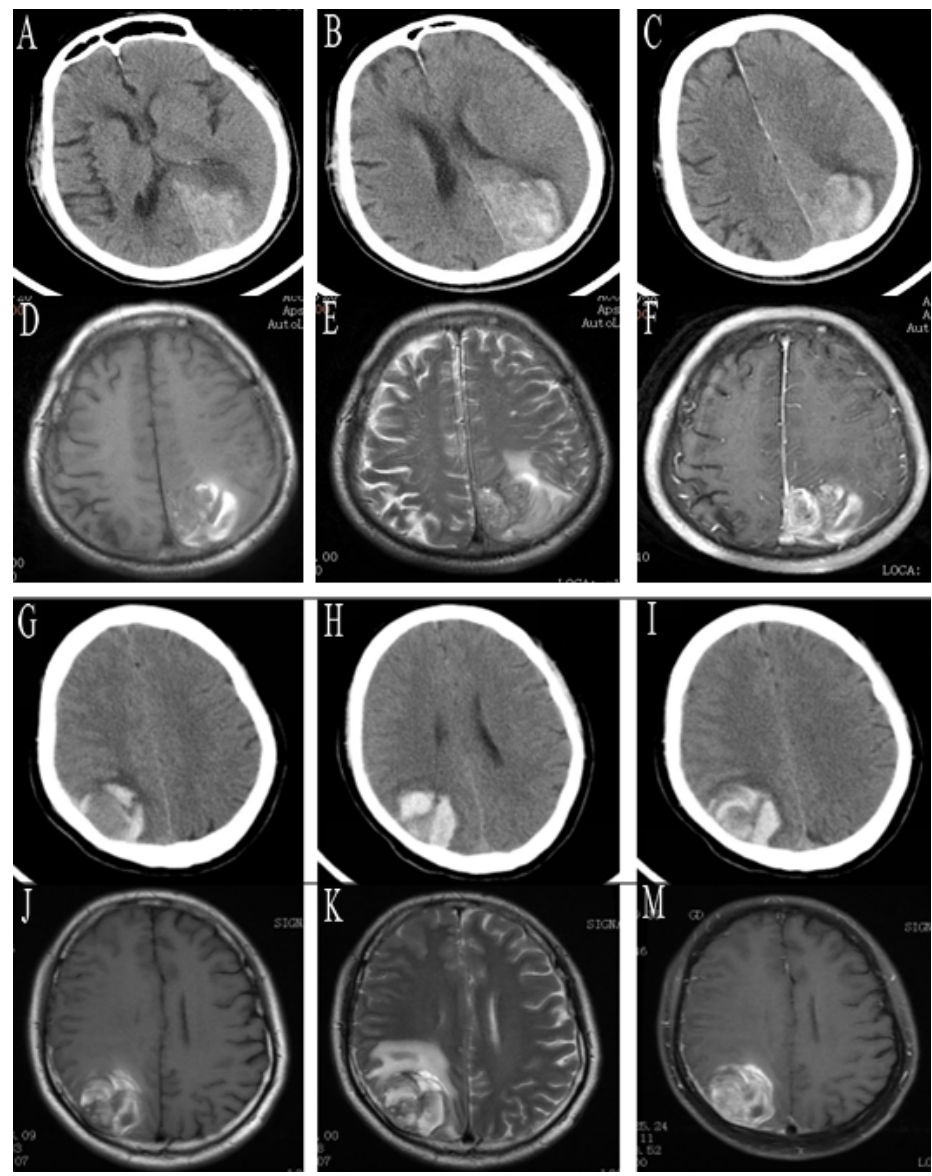

Figure 5

Neuroimaging outcomes on representative case 18 and case 16 with combined intra/extratumoral hemorrhage. Brain CT and MRI showing two extra-brain mass lesions in the left parasagital region (A-F) and right parietal-occipital area (G-M) respectively, which displaced the adjacent brain and had acute bleeding involving intratumoral hemorrhage, peritumoral hematoma, subarachnoid hemorrhage, and subdural hemorrhage. Two tumors were heterogeneously enhanced, and significant brain edema was visible in the adjacent brain parenchyma.

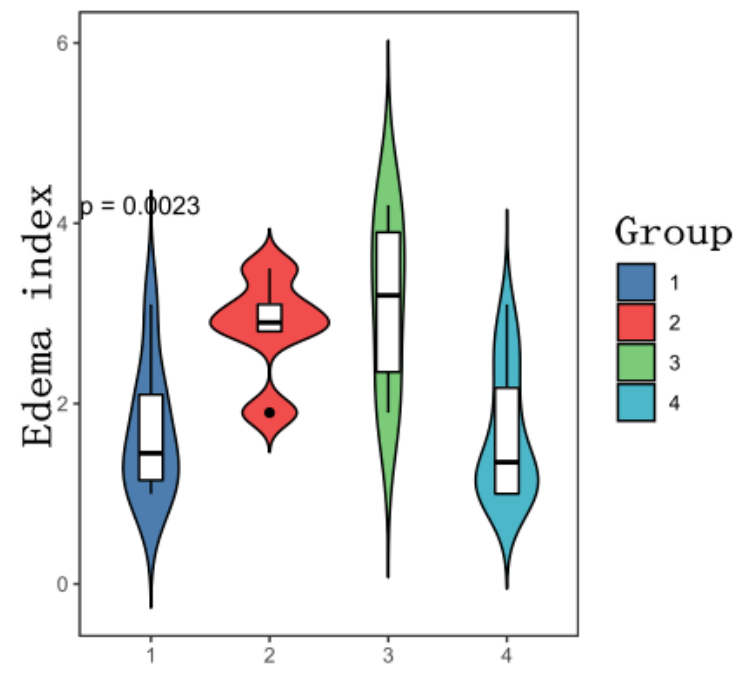

Figure 6

The graph demonstrating the difference of edema index among the four groups. The edema indexes in group 3 and group 2 were both significantly higher than those in group 1 and control group $(P<0.05)$. The edema index between group 3 and group 2 had no significant difference $(P>0.05)$; there being also no statistical difference between group 1 and control group $(P>0.05)$. 


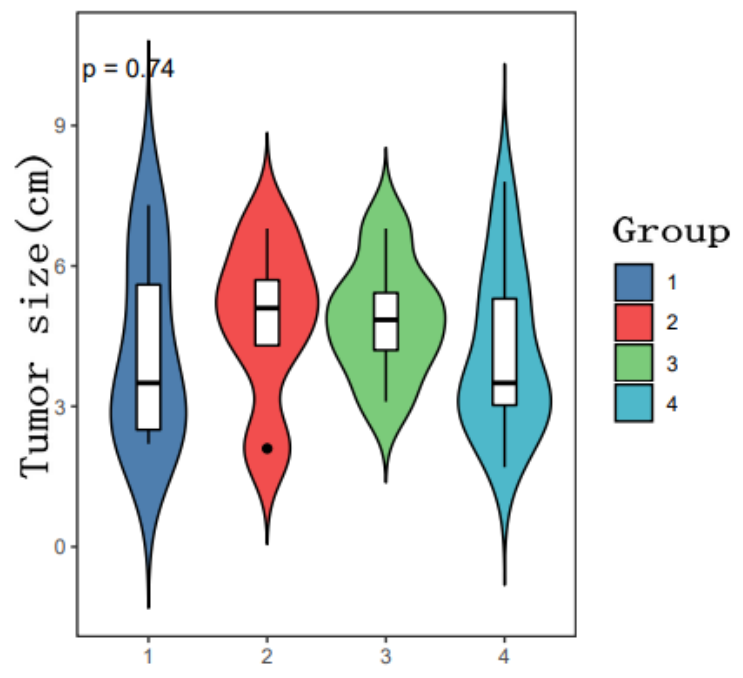

Figure 7

The graph showing no statistical difference with respect to the tumor size among the four groups $(P>0.05)$.
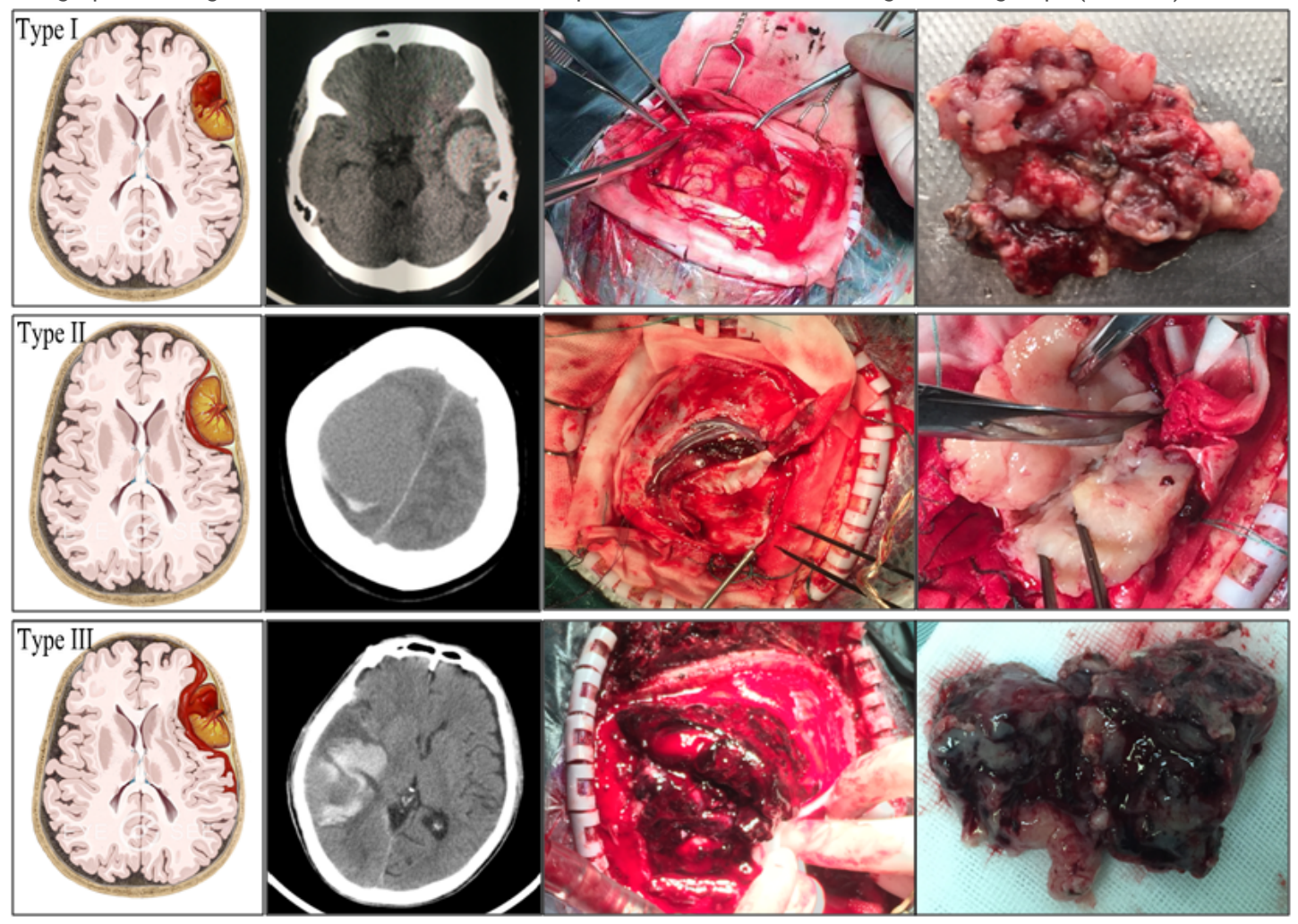

Figure 8

Operative findings associated with corresponding radiological results in different bleeding types. 